\title{
PERIODIC SOLUTIONS FOR A CLASS OF ORDINARY DIFFERENTIAL EQUATIONS
}

JAMES R. WARD, JR.

\begin{abstract}
A $T$-periodic solution to the differential equation $x^{\prime \prime}+c x^{\prime}+g(x)=$ $f(t) \equiv f(t+T)$ is shown to exist whenever a simple condition on $g$ holds, provided $c \neq 0$. No assumption is made concerning the growth of $g$. The condition on $g$ is necessary if $g$ is either an increasing or a decreasing function.
\end{abstract}

In this note we discuss existence of $T$-periodic solutions for the differential equation

$$
x^{\prime \prime}+c x^{\prime}+g(x)=f(t) \equiv f(t+T) .
$$

We assume that $g: R \rightarrow R$ is continuous, $f: R \rightarrow R$ is continuous and $T$-periodic, and $c \in R$. Here $R$ denotes the real numbers and $T>0$.

Let $m=T^{-1} \int_{0}^{T} f(t) d t$. We prove the following theorem.

THEOREM 1. Suppose the following conditions are satisfied:

(i) There is a number $r \geqslant 0$ such that $(g(x)-m) x \geqslant 0(<0)$ whenever $|x| \geqslant r$;

(ii) $c \neq 0$.

Then there is at least one T-periodic solution of (1.1).

Theorem 1 extends a result due to A. C. Lazer [2]. Lazer showed that under condition (i) there is a $T$-periodic solution for any $c \in R$ provided $g$ is sublinear; that is, provided $g(x) / x \rightarrow 0$ as $|x| \rightarrow+\infty$. Thus, we show that if $c \neq 0$ sublinearity is not needed. More recently, under the hypothesis that $c=0$, Fucik and Lovicar [1] showed that there is a $T$-periodic solution for any $T$-periodic forcing function $f$, provided $g(x) / x \rightarrow+\infty$ as $|x| \rightarrow+\infty$, thus solving an old problem. Theorem 1 includes an extension of the result of [1] to the case $c \neq 0$. We emphasize that we need no growth condition on $g$. Our proof is based on a simple Leray-Schauder argument.

In what follows we let $S=L^{2}(0, T)$ with norm $\|\cdot\|$ given by $\|u\|^{2}=\int_{0}^{T} u^{2} d t$ for $u \in S$. Let $A C$ be the functions absolutely continuous on $[0, T]$. By $H^{1}$ we will mean the space of functions $u \in A C$ with $u^{\prime} \in S\left({ }^{\prime} \equiv d / d t\right)$, with norm $|u|_{1}$ given by

$$
|u|_{1}=\max _{0<t<T}|u(t)|+\left\|u^{\prime}\right\|
$$

It is well known that $H^{1}$ is a Banach space.

Received by the editors May 10, 1979.

1980 Mathematics Subject Classification. Primary 34C15, 34C25.

Key words and phrases. Nonlinear ordinary differential equations, periodic solutions.

(C) 1980 American Mathematical Society 0002-9939/80/0000-0113/\$01.75 
Proof of Theorem 1. There is no loss of generality in supposing $m=0$, and we will so assume. We consider the case $(g(x)-m) x>0$ if $|x|>r$. The proof in the other case is essentially the same.

Let $L: D(L) \subseteq H^{1} \rightarrow S$ be defined as follows:

$$
D(L)=\left\{u \in H^{1}: u^{\prime} \in A C, u^{\prime \prime} \in S, u(0)=u(T), u^{\prime}(0)=u^{\prime}(T)\right\}
$$

$L u=u^{\prime \prime}$ for $u \in D(L)$.

Choose $b>0$ with $b \neq 4 n^{2} \pi^{2} / T^{2}$ for any integer $n$. Then $(L+b I)^{-1}$ exists and maps $S$ into $H^{1}$ compactly. There is a $T$-periodic solution $x$ of (1.1) if and only if the restriction of $x$ to $[0, T]$ is a solution to

$$
x=(L+b I)^{-1}\left(f+b x-g(x)-c x^{\prime}\right) .
$$

The mapping $x \rightarrow N x \equiv f+b x-g(x)-c x^{\prime}$ maps $H^{1}$ into $S$ continuously, and thus the mapping $x \rightarrow(L+b I)^{-1} N x$ is a completely continuous self map of $H^{1}$. By the Leray-Schauder degree theory (see, e.g., Lloyd [3]) there is a solution to the equation $x=(L+b I)^{-1} N x$ if there is an a priori bound in $H^{1}$ on the possible solutions to the family of equations

$$
x=\lambda(L+b I)^{-1} N x, \quad 0<\lambda<1 .
$$

It is easily seen that $x \in H^{1}$ is a solution of (1.3) if and only if $x \in D(L)$ and $x$ extends on $R$ to a $T$-periodic solution of the equation

$$
x^{\prime \prime}+(1-\lambda) b x+\lambda c x^{\prime}+\lambda g(x)=\lambda f .
$$

Suppose $x$ is a $T$-periodic solution of (1.4). Multiplying each side of (1.4) by $x^{\prime}$, integrating the resulting equation from 0 to $T$, and using $x(0)=x(T), x^{\prime}(0)=$ $x^{\prime}(T)$, we obtain

$$
\lambda \int_{0}^{T} c\left|x^{\prime}\right|^{2} d t=\lambda \int_{0}^{T} f x^{\prime} d t
$$

Thus, for $0<\lambda<1$, we have

$$
\left\|x^{\prime}\right\| \leqslant\|f\| /|c| \text {. }
$$

Now integrating each side of equation (1.4) from 0 to $T$ we obtain

$$
(1-\lambda) b \int_{0}^{T} x(t) d t+\lambda \int_{0}^{T} g(x(t)) d t=0,
$$

since $m=0$. By the mean value theorem for integrals, there is a number $z$, $0<z \leqslant T$, such that

$$
(1-\lambda) b x(z)+\lambda g(x(z))=0 .
$$

Since $b>0$ and $g(x) x \geqslant 0$ if $|x| \geqslant r,(1.6)$ implies that $|x(z)|<r$. We now may write

$$
x(t)=x(z)+\int_{z}^{t} x^{\prime}(s) d s
$$

and thus

$$
|x(t)| \leqslant r+T^{1 / 2}\left\|x^{\prime}\right\|
$$

by the Cauchy-Schwarz inequality. Combining (1.5) and (1.7) we have, for any solution $x$ and any $\lambda, 0<\lambda<1$, 


$$
|x|_{1} \leqslant r+T^{1 / 2}\left\|x^{\prime}\right\|+\left\|x^{\prime}\right\| \leqslant r+\left(T^{1 / 2}+1\right)\|f\| /|c| .
$$

By (1.8) all possible solutions of (1.3) are bounded in $H^{1}$, independently of $\lambda$, $0<\lambda<1$. The existence of a $T$-periodic solution follows by the Leray-Schauder alternative, as indicated earlier. Q.E.D.

Remarks. Let $h: R \rightarrow R$ be continuous. Then a minor modification in the proof of Theorem 1 can be used to show that the equation

$$
x^{\prime \prime}+h(x) x^{\prime}+g(x)=f(t) \equiv f(t+T)
$$

has a $T$-periodic solution provided (i) holds and there is a number $c>0$ such that either $h(x) \geqslant c$ for all $x \in R$ or $h(x) \leqslant-c$ for all $x \in R$.

It is easy to verify that (i) is also necessary if $g$ is an increasing (decreasing) function. Integrate (1.9) from 0 to $T$, apply the mean value theorem for integrals and then use the assumption that $g$ is increasing (decreasing).

NOTE ADDED IN PROOF. Shortly before proofs for this paper were received a paper of J. Bebernes and M. Martelli, Periodic solutions for Liénard systems, appeared in Bol. Un. Mat. Ital. (5) 16-A (1979), 398-405. They obtain the same results by a different (and slightly longer) method.

\section{REFERENCES}

1. S. Fucik and V. Lovicar, Periodic solutions of the equation $x^{\prime \prime}(t)+g(x(t))=p(t)$, Casopis Pèst. Mat. 100 (1975), 160-175.

2. A. C. Lazer, On Schauder's fixed point theorem and forced second-order nonlinear oscillations, J. Math. Anal. Appl. 21 (1968), 421-425.

3. N. G. Lloyd, Degree theory, Cambridge Univ. Press, Cambridge, 1978.

Department of Mathematics, Pan American University, Edinburg, Texas 78539

Current address: Department of Mathematics, University of Alabama, University, Alabama 35486 